\title{
COMBINATORIAL EQUIVALENCE VERSUS TOPOLOGICAL EQUIVALENCE
}

\author{
BY \\ BARRY MAZUR
}

In [8], Milnor exhibited an example of two finite complexes $X_{1}, X_{2}$ which are homeomorphic, but combinatorially distinct. That was the first example of the disparity between combinatorial notions versus topological ones on finite complexes. Previously the only results were positive in nature. Papakyriakopoulos had proved the Hauptvermutung for 2-dimensional complexes [11], and Moise [10] (and later Bing) had proved the Hauptvermutung for 3-dimensional manifolds. The object of this paper is to provide an example (see \$5) of a finite complex $K$ and two simplicial imbeddings, $\alpha, \beta: K \rightarrow S^{m}$ which are combinatorially inequivalent, yet topologically equivalent. The nature of the argument is such that the minimum $m$ it yields is $m=23$.

The construction of this example uses relative forms of the strong and weak stability theorem (see [6]). It also uses recent results of Haefliger, Bott, and Milnor. To show that the two imbeddings $\alpha, \beta: K \rightarrow S^{m}$ are not combinatorially equivalent, I notice that

$$
\begin{aligned}
& S^{m} / \alpha(K) \approx X_{1}, \\
& S^{m} / \beta(K) \approx X_{2}
\end{aligned}
$$

(the isomorphisms being combinatorial). Since $X_{1}$ is combinatorially distinct from $X_{2}$, the result follows that there is no combinatorial homeomorphism $\kappa: S^{m} \approx S^{m}$ such that $\kappa \circ \alpha=\beta$.

In the course of the construction, a general theorem is proved ( $\$ 6)$ which provides the possibility of producing many pairs of (combinatorially distinct) combinatorial imbeddings which are topologically equivalent.

An unsettled question is the following:

Are there combinatorial imbeddings $f, g: S^{k} \rightarrow S^{m}$ which are combinatorially distinct yet topologically equivalent?

By recent results of Zeeman [17], all combinatorial imbeddings of $S^{k}$ in $S^{m}(m \geqq k+3)$ are equivalent combinatorially. By the results of Smale [12], all combinatorial imbeddings of $S^{m-1}$ in $S^{m}$ which are combinatorially locally trivial are combinatorially equivalent (for $m$ sufficiently large). It is yet possible, however, for there to be two combinatorial imbeddings

Received by the editors February 20, 1962. 


$$
f, g: S^{m-2} \rightarrow S^{m}
$$

which are topologically equivalent but not combinatorially so. See the recent work of Stallings [13] related to this question, also.

A weaker question may be asked, which, in the light of Theorem 6.1, is relevant.

Are there two combinatorial imbeddings $f, g: S^{m-2} \rightarrow S^{m}$ such that these bounded complements $M_{f}, M_{g} \subseteq S^{\prime n}$ have the same homotopy type but distinct simple homotopy type?

Finally, let me point out a relation between the main construction of this paper and the very strong piecewise linear approximation theorems of Moise and Bing in three dimensions.

Let us consider the following homeomorphism approximation statement for n-dimensional euclidean space:

$\left(\mathrm{App}_{n}\right)$ Let $U \subset R^{n}$ be an open set and $K \subset U$ a closed piecewise linear subcomplex. Let $f: U \rightarrow R^{n}$ be a topological homeomorphism which is piecewise linear on $K$. Let $\varepsilon(x)>0$ be a continuous function on $U$.

Then there is a piecewise linear homeomorphism $g: U \rightarrow R^{n}$ which agrees with $f$ on the subcomplex $K$, and such that

$$
|f(x)-g(x)|<\varepsilon(x)
$$

for all $x \in U$.

This approximation statement would imply the triangulation theorem and the Hauptvermutung for $n$ dimensions. In fact, Moise and Bing prove exactly this theorem in dimension three, from which their classical results follow [10, IV; 0, Theorem 4, p. 149]. Actually, the triangulation theorem and Hauptvermutung is also implied by a significantly weaker theorem than $\mathrm{App}_{n}$. (See Theorem 3 of [0].)

The absolute version of $\mathrm{App}_{n}$ (i.e., where $K$ is empty) has recently been proven by Connell under the additional hypotheses that the map $f$ be stable, and $n \geqq 7$. See [18].

From the main construction of this paper, one may show:

Proposition. There is an integer $n$ for which $\mathrm{App}_{n}$ is false.

In fact, we may take $n=23$. Also, $\mathrm{App}_{n}$ is false in the following way: There is a closed subcomplex $K \subset R^{n}$ and a homeomorphism which is piecewise linear on $K$ such that there just is no piecewise linear homeomorphism $g$, on $R^{n}$, which agrees with $f$ on $K$.

0 . Terminology and conventions. $R^{n}$ is euclidean $n$-space with usual linear norm, $D^{n} \subseteq R^{n}$ is given by

$$
D^{n}=\left\{\chi \in R^{n} \mid\|\chi\| \leqq 1\right\} . \quad S^{n}=\partial D^{n+1}=\left\{\chi \in R^{n+1} \mid\|\chi\|=1\right\} .
$$

The word "combinatorial" means simplicial after some subdivision. Thus a 
map $f: K \rightarrow L$ is combinatorial if it is simplicial for some subdivisions $K^{\prime}, L^{\prime}$ of $K, L$, respectively. Two complexes $K, L$ are combinatorially equivalent (denoted $K \approx L$ ) if they have rectilinear subdivisions $K^{\prime}, L^{\prime}$ which are isomorphic (simplicially, of course).

If $X$ is a finite complex, $Y \subseteq X$ a subcomplex, then $X / Y$ is the quotient topological space of $X$, with the set $Y$ identified to a point. The set $X / Y$ may be again considered as a finite complex (unique up to combinatorial equivalence) in a natural way.

Namely, let $X^{\prime}$ be the first barycentric derived complex of $X, \Sigma \subset X^{\prime}$ the simplicial neighborhood of $Y^{\prime}$ in $X^{\prime}, F \subset \Sigma$, the frontier of $\Sigma$ (i.e., the subcomplex of $\Sigma$ consisting in all simplices which do not touch $\left.Y^{\prime} \subset \Sigma\right)$.

Define the quotient simplicial complex: $X / Y=\left\{X^{\prime}-\Sigma\right\} \cup C F$.

In the above construction, $C F$ denotes the cone over $F$. This gives an explicit triangulation of the topological space $X / Y$. It should be remarked, however, that the quotient map

$$
\xi: X \rightarrow X / Y
$$

is not necessarily combinatorial!

One does have the following naturality property:

LEMMA 0.1. If $Z \subset Y \subset X$ are inclusions of simplicial complexes, then $Y / Z$ may be regarded as a subcomplex of $X / Z$, in a natural way, and

$$
(X / Z) /(Y / Z)
$$

is combinatorially equivalent to $X / Y$.

Proof. Let $\Sigma(A, B)$ (and $\mathrm{F}(A, B)$ ) denote the simplicial neighborhood (and its frontier) of $B \subset A$. Then we may write:

$$
A / B=\left\{A^{\prime}-\Sigma\left(A^{\prime}, B^{\prime}\right)\right\} \cup C F(A, B)
$$

Lemma (0.1) follows from the following two formulas:

(i) $\mathrm{F}(X / Z, Y / Z)=\mathrm{F}\left(X^{\prime}, Y^{\prime}\right)$,

(ii) $X / Z-\Sigma(X / Z, Y / Z)=X^{\prime}-\Sigma\left(X^{\prime}, Y^{\prime}\right)$.

The symbol $(\approx)$ will denote isomorphism, either topological, combinatorial, or differentiable. The context will always make clear which of the three genres is meant. If $f, g$ are maps, then $f \approx g$ means that $f$ is isotopic to $g$ (again the context will make the nature of the isotopy clear).

The symbol $(\sim)$ will denote homotopy equivalence. For maps $f, g, f \sim g$ means that $f$ is homotopic to $g$.

By a pair $(X, Y)$ is meant a space $X$ and a subspace $Y \subseteq X$. A map $f: X, Y) \rightarrow\left(X^{\prime}, Y^{\prime}\right)$ is a map $\rightarrow: X \rightarrow X^{\prime}$ such that $f(Y) \subseteq Y^{\prime}$. To say that $f_{1} \sim f_{2}\left(\right.$ or $\left.f_{1} \approx f_{2}\right)$ as mapping 


$$
f_{1}, f_{2}:(X, Y) \rightarrow\left(X^{\prime}, Y^{\prime}\right)
$$

it is meant that there is a homotopy (an isotopy)

$$
f_{t}:(X, Y) \rightarrow\left(X^{\prime}, Y^{\prime}\right)
$$

for $1 \leqq t \leqq 2$

The notation $(X, Y) \times Z$ will be used for the pair $(X \times Z, Y \times Z)$.

Manifold means $C^{\infty}$ differentiable manifold. By a manifold pair $(M, W), \mathrm{I}$ shall always mean the pair consisting of a manifold $M$ and the submanifold $W \subseteq M, W=\partial M$ (the boundary of $M$ ).

If $\phi:(M, W) \rightarrow\left(M^{\prime}, W^{\prime}\right)$ is a map of manifold pairs, the map $\partial \phi: W \rightarrow W$ will denote the restriction

$$
\partial \phi=\phi \mid W .
$$

The identity map of $X$ will be denoted

$$
1: X \rightarrow X
$$

Given a compact manifold $M$, there is a (smooth) $C^{1}$-triangulation of $M$, endowing $M$ with the structure of a finite simplicial complex $K$ (see [15]). The combinatorial structure of $K$ is uniquely determined by the manifold $M$, and will be called the underlying combinatorial structure of $M$. If $M$ is a manifold, without any special notice I will apply combinatorial notions to $M$, in which case it should be immediately assumed that I am referring to the unique combinatorial structure of $M$. (For example, we may talk of the simple homotopy type of $M$.)

For the definition and theory of simple homotopy type, see [14] and also [8]. For the foundations of differential topology, see $[16 ; 9]$.

In particular, I shall assume known the Tubular Neighborhood Lemma (due to Thom) in [9], and the general position techniques, the Whitney imbedding theorem, etc., originating in [16]. There are many modern treatments of this theory to be found. (For example, [9].)

For classical homotopy-theoretic concepts (i.e., Serre fibre spaces, calculations of the first few stable homotopy groups of spheres, etc.) refer to $\mathrm{Hu}, \mathrm{Homo}-$ topy theory, Academic Press, 1960.

CX will denote the cone of $X, \Omega X$ will denote the loopspace of $X$.

1. The functor $K^{*}$. Let $(A, B)$ be a pair of spaces. Then one may define

$$
K^{-i}(A, B)=\left[(A, B) ;\left(\Omega^{i} S O, \varepsilon_{i}\right)\right]
$$

where $\varepsilon_{i}$ is the unit element of the $H$-space $\Omega^{i} S O$. By virtue of the Bott periodicity results $[1], K^{i}(A, B)$ is defined for both positive and negative exponents, $i$, and 
"periodic" of order 8. If $a \in A$ is a base point, abbreviate $K^{i}(A, a)$ by $K^{i}(A)$. Since the $\Omega^{i} S O$ are homotopy abelian groups, each $K^{i}(A, B)$ may be given the structure of an abelian group $\left({ }^{1}\right)$.

If $f:\left(A_{1}, B_{1}\right) \rightarrow\left(A_{2}, B_{2}\right)$ is a map, there is induced a homomorphism $K^{i}(f): K^{i}\left(A_{2}, B_{2}\right) \rightarrow K^{i}\left(A_{1}, B_{1}\right)$ in an obvious way. Thus $K^{*}$ may be regarded as a contravariant functor of the category of pairs of topological spaces into the category of abelian groups.

The functor $K^{*}$ is a cohomological functor. Explicitly, it obeys the excision axiom for cohomology, and if $(A, B)$ is a topological pair, there are natural maps

$$
\delta^{i}: K^{i}(B) \rightarrow K^{i+1}(A, B)
$$

giving rise to long exact sequences:

$$
\cdots \rightarrow K^{i}(A) \rightarrow K^{i}(B) \stackrel{\delta^{i}}{\rightarrow} K^{i+1}(A, B) \rightarrow K^{i+1}(A) \rightarrow \cdots
$$

for all $i$.

For our later purposes, we shall need notation for a group, fairly unnatural to this setting. If $(A, B)$ is a pair, let $k(A, B)$ be the quotient group of $K^{\circ}(B)$ modulo the image of $K^{0}(A)$. That is:

$$
K^{0}(A) \rightarrow K^{0}(B) \rightarrow k(A, B) \rightarrow 0
$$

is exact.

Proposition 1.1. Let $n \equiv 3,5,6,7(\bmod 8)$. Let $A, B$ be bounded submanifolds of $S^{n}$ such that

(i) $A \cup B=S^{n}$,

(ii) $A \cap B=W$

where $W$ is the common boundary of $A$ and $B$,

$$
W=\partial A=\partial B \text {. }
$$

Let the following diagram be composed of the natural maps:

$$
\begin{gathered}
K^{0}(A) \rightarrow K^{0}(W) \rightarrow k(A, W) \rightarrow 0 . \\
\bigcap_{K^{0}(B)} \nearrow_{\gamma}
\end{gathered}
$$

Then $\gamma$ is surjective.

(1) At this point it should be remarked that the group structures on $K^{0}(A, B)$ coming from: (i) the group structure on $S O$, (ii) the group structure on $\Omega^{8} S O$, coincide. 
Proof. Consider the diagram:

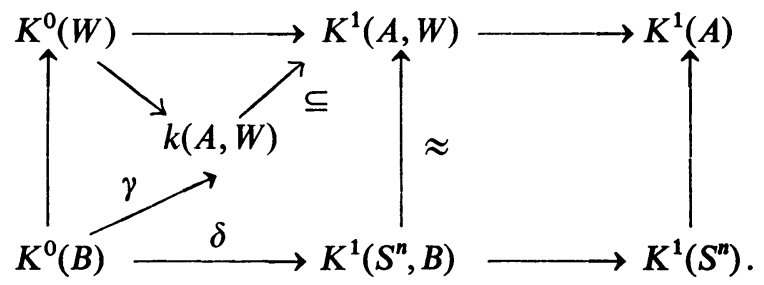

The middle vertical arrow is an isomorphism by excision. The conditions on $n$ required by (1.1) are exactly such as to insure that $K^{1}\left(S^{n}\right)=0$ after the work of Bott [1].

Consequently $\delta$ is surjective and the proposition follows.

2. Stability for pairs. The section is devoted to restating results of [6] in relativized versions particularly suited for their eventual applications.

Strong and weak stable equivalence for pairs is defined in analogy with the absolute case. If $\left(A_{i}, B_{i}\right)$ are manifold pairs $(i=1,2)$, then a diffeomorphism

$$
f:\left(A_{1}, B_{1}\right) \times D^{k} \approx\left(A_{2}, B_{2}\right) \times D^{k}
$$

is called a strong $k$-equivalence between $\left(A_{i}, B_{i}\right), i=1,2$; it is denoted

$$
f:\left(A_{1}, B_{1}\right) \underset{\underset{\sim}{(k)}}{\rightarrow}\left(A_{2}, B_{2}\right) .
$$

A diffeomorphism

$$
f:\left(A_{1}, B_{1}\right) \times R^{k} \rightarrow\left(A_{2}, B_{2}\right) \times R^{k}
$$

is called a weak $k$-equivalence and is denoted

$$
f:\left(A_{1}, B_{1}\right) \underset{\sim}{(\vec{\sim})}\left(A_{2}, B_{2}\right) .
$$

A $k$-equivalence

$$
f:\left(A_{1}, B_{1}\right) \underset{(k)}{\rightarrow}\left(A_{2}, B_{2}\right)
$$

is said to be homotopic to a continuous map $f:\left(A_{1}, B_{1}\right) \rightarrow\left(A_{2}, B_{2}\right)$ if the following diagram is homotopy commutative:

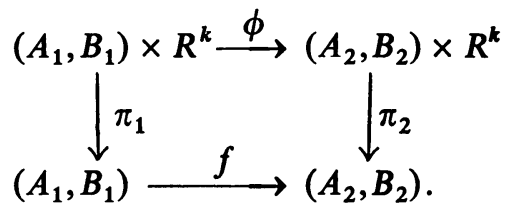

Let $\tau(M)$ denote the stable vector bundle class of the tangent bundle of $M$, when $M$ is a manifold. Then $\tau(M)$ may be considered to be an element $\tau(M) \in K^{+1}(M)$. 
If $f: M \rightarrow M^{\prime}$ is a continuous map, then $I$ will denote the map

$$
K^{+1}(f): K^{+1}\left(M^{\prime}\right) \rightarrow K^{+1}(M)
$$

by the symbol $f^{*}=K^{+1}(f)$.

Let $f:\left(A_{1}, B_{1}\right) \rightarrow\left(A_{2}, B_{2}\right)$ be a simple homotopy equivalence between manifold pairs. Then $f$ is called a simple tangential equivalence if

(i) $f^{*} \tau\left(A_{2}\right)=\tau\left(A_{1}\right)$,

(ii) $f^{*} \tau\left(B_{2}\right)=\tau\left(B_{1}\right)$.

STrong StABility THEOREM FOR PAIRS. If $f:\left(A_{1}, B_{1}\right) \rightarrow\left(A_{2}, B_{2}\right)$ is a simple tangential equivalence between compact $n$-dimensional manifold pairs, then for any $k \geqq n+1$, there exists a strong k-equivalence

$$
\phi:\left(A_{1}, B_{1}\right) \underset{(\vec{k})}{\underset{\approx}{\rightarrow}}\left(A_{2}, B_{2}\right)
$$

such that $\phi$ is homotopic to $f$ as a mapping of the pair $\left(A_{1}, B_{1}\right)$ onto $\left(A_{2}, B_{2}\right)$. Since $\phi$ is a diffeomorphism, from the above it follows that $\phi$ induces a map of pairs:

$$
\phi:\left(A_{1} \times D^{k}, A_{1} \times \partial D^{k}\right) \underset{\approx}{\underset{ }{2}}\left(A_{2} \times D^{k}, A_{2} \times \partial D^{k}\right) .
$$

The diffeomorphism $\phi$ may also be chosen so that $\phi \sim f \times 1_{k}$ where the maps $\phi, f \times 1_{k}$ are taken on the pair $\left(A_{1} \times D^{k}, A_{1} \times \partial D^{k}\right)$.

REMARK. The "natural" stable range for this last statement to be true is: $k \geqq n+2$. A further argument shows that it holds for $k \geqq n+1$ as well.

The above theorem, in absolute version, is stated in [6]. A proof of it is given in [7], and also, independently, it has been proved by Hirsch in an unpublished paper. It is quite elementary to deduce the relative version above from the absolute result, by employing standard (stable) differential-topological arguments.

WEAK STABILITY THEOREM FOR PAIRS. If $f:\left(A_{1}, B_{1}\right)$ is a tangential equivalence between compact $n$-dimensional manifold pairs, then for any $k \geqq n+1$, there exists a weak $k$-equivalence

$$
\phi:\left(A_{1}, B_{1}\right) \underset{\sim}{(k)}\left(A_{2}, B_{2}\right)
$$

such that $\phi$ is homotopic to $f$.

This is not as easily obtainable from the statement of its absolute counterpart. In absolute version it was reported in [6]; also, a sketch of the proof was given there. The Bourbaki report [5] of Serge Lang's contains a more detailed account of the proof.

This theorem will be proved in a subsequent paper, its proof being quite analogous to the absolute version. 
The point of the above two theorems is that simple tangential equivalences and tangential equivalence are homotopy conditions. That is, they are essentially algebraic prescriptions on the manifold pairs, whereas weak and strong $k$-equivalence are differential-topological concepts. Thus in both of the above theorems, algebraic hypotheses imply differential-topological conclusions. For purposes of application, I must rephrase the weak stability theorem somewhat. The remainder of this section is devoted to paraphrasing the theorem so as to make it more suitable for our uses.

If $\left(A_{1}, B_{1}\right)$ is a pair, denote by $\beta:\left(A_{1}, B_{1}\right) \rightarrow\left(A_{1}, B_{1}\right) \times R^{k}$ the zero-section map, and by

$$
\pi:\left(A_{1}, B_{1}\right) \times R^{k} \rightarrow\left(A_{1}, B_{1}\right)
$$

the natural projection.

If $f:\left(A_{1}, B_{1}\right) \times R^{k} \rightarrow\left(A_{2}, B_{2}\right) \times R^{k}$ is a continuous map, then

$$
f^{\prime}=\pi \circ f \circ \beta:\left(A_{1}, B_{1}\right) \rightarrow\left(A_{2}, B_{2}\right)
$$

is also continuous. Notice that $f$ is a (simple) tangential equivalence between the $\left(A_{i}, B_{i}\right) \times R^{k}(i=1,2)$ if and only if $f^{\prime}$ is a (simple) tangential equivalence between the $\left(A_{i}, B_{i}\right)$.

Consider the following conditions $(\partial)$ one may place on a continuous map

$$
f:\left(A_{1}, B_{1}\right) \times R^{p} \rightarrow\left(A_{2}, B_{2}\right) \times R^{p}:
$$

$\left(\partial_{1}\right) f$ is a tangential equivalence,

$\left(\partial_{2}\right)$ if $\partial f=f \mid B_{1} \times R^{p}$, then $\partial f$ is a diffeomorphism,

$$
\partial f: B_{1} \times R^{p} \rightarrow B_{2} \times R^{p} .
$$

Given a continuous map

$$
f:\left(A_{1}, B_{1}\right) \times R^{p} \rightarrow\left(A_{2}, B_{2}\right) \times R^{p}
$$

satisfying $(\partial)$, I should like to find (in the spirit of the weak stability theorem a weak $p$-equivalence

$$
\phi:\left(A_{1}, B_{1}\right) \underset{\sim}{\underset{\sim}{\sim}}\left(A_{2}, B_{2}\right)
$$

such that if $\partial \phi=\phi \mid B_{1} \times R^{p}$,

(i) $\partial f=\partial \phi$,

(ii) $f$ is homotopic to $\phi$.

This is not always possible, but there is a fairly obvious "obstruction" to its possibility.

Let, then, $f$ satisfy $(\partial)$. I shall define a unique element

$$
k(f) \in k\left(A_{1}, B_{1}\right) .
$$


Let $O_{p} \in R^{p}$ denote the zero-element. Since $p$ is assumed sufficiently large with respect to $\operatorname{dim} A_{1}\left(p>\operatorname{dim} A_{1}\right)$, there is an imbedding $\phi_{0}=A_{1} \times O_{p} \rightarrow A_{2} \times R^{p}$ such that

(a) $\phi_{0}\left|B_{1} \times O_{p}=f\right| B_{1} \times O_{p}$,

(b) $\phi_{0}\left(A_{1} \times O_{p}\right) \cap B_{2} \times R^{p}=\phi_{0}\left(B_{1} \times O_{p}\right)$

and the intersection is transversal,

(c) the normal bundle to $A_{1} \times O_{p}$ as embedded by $\phi_{0}$ in $A_{2} \times R^{p}$ is trivial. Moreover, $\phi_{0}$ is unique up to isotopy.

Choose, trivializations of the normal bundles $v_{1}, v_{2}$ of $A_{1} \times O_{p}$ as imbedded (respectively) in $A_{1} \times R^{p}$ by the identity imbedding, and in $A_{2} \times R^{p}$ by $\phi_{0}$. With respect to these two trivializations, $\partial f$, restricted to $v_{1} \mid B_{1} \times O_{p}$, induces a linear bundle map:

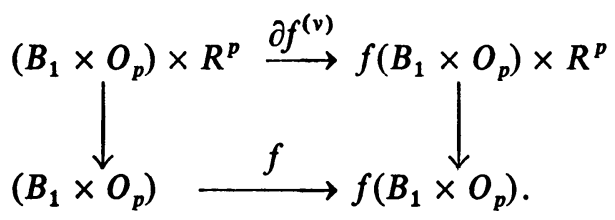

Such a bundle map yields, naturally, a homotopy class of maps

$$
\left[\partial f^{(v)}\right] \in\left[B_{1} \times O_{p} ; S O_{p}\right] \approx K^{o}\left(B_{1}\right) .
$$

(This may be seen because

$$
\partial f^{(v)}:\left(B_{1} \times O_{p}\right) \times R^{p} \rightarrow f\left(B_{1} \times O_{p}\right) \times R^{p}
$$

may be given by

$$
\partial f^{(v)}(b, \rho)=\left(f(b), \lambda_{b}(\rho)\right)
$$

with $\lambda_{b} \in G L_{p}$.)

Thus $\partial f^{(v)}$ determines a map $\overline{\partial f^{(v)}}: B_{1} \rightarrow G L_{p}$ given by

$$
\overline{\partial f^{(v)}}(b)=\lambda_{b} \text {. }
$$

Since $\left[B_{1}, S O_{p}\right] \approx\left[B_{1}, G L_{p}\right]$ we take $\left[\partial f^{(v)}\right]$ to be the homotopy class in $\left[B_{1}, S O_{p}\right]$ determined by the homotopy class of $\overline{\partial f^{(v)}}$ in $\left[B_{1}, G L_{p}\right]$. The element $\left[\partial f^{(v)}\right]$ clearly depends on the trivialization of $v_{1}$, chosen, and so the homotopy class $\left[\partial f^{(v)}\right]$ may vary by elements in the image of $K^{o}\left(A_{1}\right)$ as one varies the choice of trivialization. Thus $\left[\partial f^{\nu}\right]$ defines a unique element

$$
k(f) \in k\left(A_{1}, B_{1}\right)=K^{o}\left(B_{1}\right) / \operatorname{im} K^{o}\left(A_{1}\right) .
$$

WEAK EXTENSION THEOREM. Let $f:\left(A_{1}, B_{1}\right) \times R^{p} \rightarrow\left(A_{2}, B_{2}\right) \times R^{p}$ be a continuous map satisfying $(\partial)$; i.e., it is a tangential equivalence, and $\partial f=f \mid B_{1} \times R^{p}$ is a diffeomorphism.,

$$
\partial f: B_{1} \times R^{p} \underset{\approx}{\approx} B_{2} \times R^{p} .
$$


Then there is a diffeomorphism

$$
\phi:\left(A_{1}, B_{1}\right) \times R^{p} \underset{\approx}{\approx}\left(A_{2}, B_{2}\right) \times R^{p}
$$

such that

(a) $\phi$ is homotopic to $f$,

(b) if $\partial \phi=\phi \mid B_{1} \times R^{p}$, then

$$
\partial \phi=\partial f
$$

if and only if

$\left(\partial_{3}\right) k(f)=O \in k\left(A_{1}, B_{1}\right)$.

Proof. $k(f)=O$ is precisely the condition necessary to be able to extend the diffeomorphism

$$
\phi_{0}: A_{1} \times\left\{O_{p}\right\} \rightarrow A_{2} \times R^{p}
$$

to some tubular neighborhood,

$$
A_{1} \times D^{p} \text { of } A_{1} \times\left\{O_{p}\right\} \text { in } A_{1} \times R^{p} .
$$

Once this extension is made, the proof of the weak stability theorem allows one to find a $p$-equivalence between the $\left(A_{i}, B_{i}\right) \times R^{p}$ extending the $p$-equivalence already given between the boundaries $B_{i} \times R^{p}(i=1,2)$.

3. Reduced suspension. The various brands of suspension which are only technically distinct in algebraic topology are crucially different in terms of combinatorial structures and therefore the reader is cautioned not to confuse them.

The kind of suspension which we will study is called reduced suspension.

Definition 3.1. Let $X$ be a finite complex, $\chi_{0} \in X$ a vertex. Then the $k$ th reduced suspension of $\left(X, \chi_{0}\right)$, denoted $E^{k}\left(X, \chi_{0}\right)$, will be the topological space

$$
X \times D^{k} / X \times \partial D^{k} \cup \chi_{0} \times D^{k}
$$

endowed with the quotient combinatorial structure. The point to which the set $X \times \partial D^{k} \cup \chi_{0} \times D^{k}$ has been identified will be denoted $\chi_{k} \in E^{k}(X, \chi)$ (the base point of $\left.E^{k}\left(X, \chi_{0}\right)\right)$.

If $X$ is a connected combinatorial manifold, its base point $\chi \in X$ will always be chosen interior to $X$ and clearly $E^{k}(X, \chi)$ is independent of the choice of $\chi \in$ int $X$. It will be denoted $E^{k}(X)$. Notice that $E^{k}(X, \chi)-\left\{\chi_{k}\right\} \approx(X-\{\chi\}) \times$ int $D^{k}$, is naturally a differentiable manifold, when $X$ is a differentiable manifold.

A relative differentiable manifold $(A, B)$ will denote a pair of simplicial complexes $(A, B)$ such that the space $A-B$ is endowed with the structure of a differentiable manifold with respect to which the decomposition induced by the simplicial structure of $A$ is a smooth $\left(C^{1}\right)$ open triangulation. Thus, $\left(E^{k}(X, \chi), \chi_{k}\right)$ is a relative differentiable manifold pair. 
A differentiable map

$$
f:(A, B) \rightarrow\left(A^{\prime}, B^{\prime}\right)
$$

of relative differentiable manifold pairs is a piecewise differentiable map $f:(A, B) \rightarrow\left(A^{\prime}, B^{\prime}\right)$ such that $f \mid A-B$ is differentiable.

The notions of homotopy, isomorphism, isotopy, etc., for differentiable manifold pairs are defined in the obvious way.

If $f:(X, \chi) \rightarrow(Y, y)$ is a combinatorial map, there is a natural map $E^{k}(f): E^{k}(X, \chi) \rightarrow E^{k}(Y, y)$ induced by the above construction of reduced suspension.

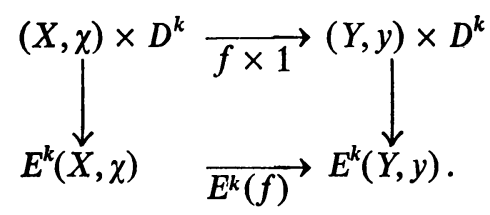

If $f:(X, \chi) \rightarrow(Y, y)$ is a differentiable map of differentiable manifolds, then

$$
E^{k}(f):\left\{E^{k}(x, \chi), \chi_{k}\right\} \rightarrow\left\{E^{k}(Y, y), y_{k}\right\}
$$

is a differentiable map of relative differentiable manifold pairs.

The assignation $E^{k}$ for maps and objects is clearly a covariant functor.

For compactness of notation, I shall introduce the term isotopy-action to describe actions of groups on spaces unique up to isotopy.

Definition 3.2. Let $(X, Y)$ be a simplicial complex pair, a manifold pair, or a relative differentiable manifold pair. Let

$$
\eta: \operatorname{Aut}(X, Y) \rightarrow \text { Is. } \operatorname{Aut}(X, Y)
$$

be the natural map of the group of automorphisms of $(X, Y)$ onto the group of isotopy classes of automorphisms of $(X, Y)$. Let $\Phi: G \rightarrow \operatorname{Aut}(X, Y)$ be a map of an abstract group $G$ into $\operatorname{Aut}(X, Y)$. Then $\Phi$ will be called an isotopy-action of $G$ on $(X, Y)$ if $\eta \Phi: G \rightarrow$ Is. Aut $(X, Y)$ is a homomorphism of groups.

Let $X$ be a differentiable manifold, $k>\operatorname{dim} X$, and $E^{k}(X, x)$ the reduced suspension of $(X, x)$.

Since $k$ is large enough, there is an isomorphism $\mu: K^{0}(X) \underset{\approx}{\longrightarrow}\left[X, S O_{k}\right]$. (See $\S 1$ for definition of $K^{0}(X)$.)

For each $\lambda \in K^{0}(X)$, let

$$
f_{\lambda}: X \rightarrow S O_{k}
$$

be a differentiable map such that $\left[f_{\lambda}\right]=\mu(\lambda)$ (i.e., $f_{\lambda}$ is a differentiable representative for the homotopy class $\lambda$ ).

Define $\phi_{\lambda}: X \times D^{k} \rightarrow X \times D^{k}$ by:

$$
\phi_{\lambda}(\chi, d)=\left(\chi, f_{\lambda}(\chi) \cdot d\right)
$$


where $\chi \in X, d \in D^{k}$ and if $g \in S O_{k}, r \in R^{k}, g \cdot r \in R^{k}$ denotes the element of $R^{k}$ obtained by applying the orthogonal transformation $g$ on the element $r \in R^{k}$.

Notice that $\phi_{\lambda}$ induces an automorphism of the pair,

$$
\left(X \times D^{k},\{\chi\} \times D^{k} \cup X \times \partial D^{k}\right) .
$$

Hence $\phi_{\lambda}$ induces an automorphism, $\Phi_{\lambda}$, of

$$
\left(E^{k}(X, \chi), \chi_{k}\right)
$$

after identification.

It is clear that if another choice, $f_{\lambda}^{\prime}$ were made as a representative of the homotopy class $\lambda, f_{\lambda}^{\prime} \sim f_{\lambda}$, the automorphism $\Phi_{\lambda}^{\prime}$ constructed would be isotopic to $\Phi_{\lambda}$.

LEMMA 3.3. The map

$$
\Phi: K^{0}(X) \rightarrow \operatorname{Aut}\left\{E^{k}(X, \chi), \chi_{k}\right\}
$$

is a differentiable isotopy-action of $K^{0}(X)$ on the relative differentiable manifold pair,

$$
\left\{E^{k}(X, \chi), \chi_{k}\right\}
$$

Proof. Immediate, granted the footnote to $\$ 1$.

If $(A, B)$ is a differentiable manifold pair, $B=\partial A$, denote by $\partial E^{k}(A)$ the natural image of $B \times D^{k}$ in $E^{k}(A)$.

$$
\begin{array}{cc}
A \times D^{k} \longrightarrow & E^{k}(A) \\
& \text { UI } \\
B \times D^{k} \longrightarrow & \text { UI } \\
& \partial E^{k}(A) .
\end{array}
$$

The crucial theorem concerning reduced suspension (for our purposes) is the following:

THEOREM 3.4. Let

$$
f:\left(A_{1}, B_{1}\right) \rightarrow\left(A_{2}, B_{2}\right)
$$

be a simple tangential equivalence between the two n-dimensional differentiable manifold pairs $\left(A_{i}, B_{i}\right)($ for $i=1,2)$.

Assume that $\left(A_{1}, B_{1}\right)$ (and hence: $\left(A_{2}, B_{2}\right)$ ) is 3-connected. Let $k=n+1$. There is a differentiable isomorphism of relative manifold pairs,

$$
\eta:\left\{E^{k}\left(A_{1}\right), \chi_{1}\right\} \underset{\approx}{\vec{a}}\left\{E^{k}\left(A_{2}\right), \chi_{2}\right\}
$$

which induces a map

$$
\eta:\left\{E^{k}\left(A_{1}\right), \partial E^{k}\left(A_{1}\right)\right\} \underset{\approx}{\longrightarrow}\left\{E^{k}\left(A_{2}\right), \partial E^{k}\left(A_{2}\right)\right\}
$$


The isomorphism $\eta$ may also be chosen so as to satisfy the following requirement:

$$
\eta \sim E^{k} f:\left\{E^{k}\left(A_{1}\right), \partial E^{k}\left(A_{1}\right)\right\} \rightarrow\left\{E^{k}\left(A_{2}\right), \partial E^{k}\left(A_{2}\right)\right\} .
$$

REMARK. For the duration of this paper, set:

$$
k=n+1 .
$$

The result holds as well for $k \geqq n+1$ as will be pointed out in the course of the proof.

Proof of Theorem 3.4. Applying the strong stability theorem (\$2) one obtains a diffeomorphism

$$
\phi:\left(A_{1}, B_{1}\right) \times D^{k} \underset{\quad}{\vec{*}}\left(A_{2}, B_{2}\right) \times D^{k} .
$$

The mapping (-1): $D^{k} \rightarrow D^{k}$ is given by the formula:

$$
(-1)\left(d_{1}, \cdots, d_{k}\right)=\left(d_{1}, \cdots, d_{k-1},-d_{k}\right)
$$

for $d=\left(d_{1}, \cdots, d_{k}\right) \in D^{k}$. By the symbol $( \pm 1)$ I mean one of the two mappings, $(-1): D^{k} \rightarrow D^{k}$ or the identity mapping, $(+1): D^{k} \rightarrow D^{k}$. In general, if $f:\left(D^{k}, \partial D^{k}\right) \rightarrow(X, Y)$ is a map, $-f$ will denote the composite,

$$
-f=f \circ(-1) \text {. }
$$

Let $a_{i} \in \operatorname{int} A_{i}$ be the base points of $A_{i}, i=1,2$.

Consider the imbeddings:

$$
\pm \phi_{2}, \phi_{1}:\left(D^{k}, \partial D^{k}\right) \rightarrow\left(A_{2} \times D^{k}, A_{2} \times \partial D^{k}\right)
$$

given by:

(1) $\left( \pm \phi_{2}\right)(d)=\left(a_{2},( \pm 1)(d)\right), d \in D^{k}$,

(2) $\phi_{1}(d)=\phi\left(a_{1}, d\right), \quad d \in D^{k}$.

LeMma 3.5. $\pm \phi_{2} \sim \phi_{1}$. That is, for $1 \leqq t \leqq 2$ there is a homotopy

$$
\psi_{t}:\left(D^{k}, \partial D^{k}\right) \rightarrow\left(A_{2} \times D^{k}, A_{2}, \times \partial D^{k}\right)
$$

such that

$$
\psi_{2}= \pm \phi_{2}, \psi_{1}=\phi_{1} .
$$

Proof. The natural imbedding

$$
c_{i}:\left(D^{k}, \partial D^{k}\right) \rightarrow\left\{a_{i}\right\} \times\left(D, \partial D^{k}\right) \subseteq A_{i} \times\left(D^{k}, \partial D^{k}\right)
$$

induces isomorphisms,

$$
Z \approx \pi_{k}\left(D^{k}, \partial D^{k}\right) \stackrel{\left(c_{i}\right)_{*}}{\underset{\sim}{\longrightarrow}} \pi_{k}\left(A_{i} \times D^{k}, A_{i} \times \partial D^{k}\right)
$$

for $i=1,2$. 
Let $\left[\phi_{2}\right],\left[\phi_{1}\right] \in \pi_{k}\left(A_{2} \times D^{k}, A_{2} \times \partial D^{k}\right) \approx Z$ be the relative homotopy classes represented by $\phi_{2}, \phi_{1}$, respectively.

To prove Lemma (3.5) it suffices to show that $\pm\left[\phi_{2}\right]=\left[\phi_{1}\right]$.

If $\zeta_{i} \in \pi_{k}\left(A_{i} \times D^{k}, A_{i} \times \partial D^{k}\right) \approx Z$ are generators, $i=1,2$, clearly

where

$$
\begin{aligned}
& {\left[\phi_{2}\right]= \pm \zeta_{2},} \\
& {\left[\phi_{1}\right]= \pm \phi_{*}\left(\zeta_{1}\right)}
\end{aligned}
$$

$$
\phi_{*}: \pi_{k}\left(A_{1} \times D^{k}, A_{1} \times \partial D^{k}\right) \underset{\approx}{\approx}\left(A_{2} \times D^{k}, A_{2} \times \partial D^{k}\right)
$$

is the isomorphism induced by the diffeomorphism $\phi$. Since $\phi_{*}$ is an isomorphism, $\phi_{*}\left(\zeta_{1}\right) \approx \pm \zeta_{2}$, and Lemma (3.5) follows.

LEMMA 3.6. The differentiable imbeddings

$$
\begin{array}{r}
\phi_{1}:\left(D^{k}, \partial D^{k}\right) \rightarrow A_{2} \times\left(D^{k}, \partial D^{k}\right), \\
\pm \phi_{2}:\left(D^{k}, \partial D^{k}\right) \rightarrow A_{2} \times\left(D^{k}, \partial D^{k}\right)
\end{array}
$$

are isotopic. That is, for $1 \leqq t \leqq 2$, there is a global isotopy,

$$
H_{t}:\left(A_{2} \times D^{k}, A_{2} \times \partial D^{k}\right) \underset{\approx}{\underset{ }{(}}\left(A_{2} \times D^{k}, A_{2} \times \partial D^{k}\right)
$$

such that $H_{1}=1$, and

$$
A_{2} \times\left(D^{k}, \partial D^{k}\right) \underset{\phi_{1}}{\stackrel{H_{2}}{\sim} \underset{\left(D^{k}, \partial D^{k}\right)}{\sim} A_{2} \times\left(D^{k}, \partial D^{k}\right)}
$$

is commutative. $\left(B y\left( \pm \phi_{2}\right)\right.$ I mean either $\left(+\phi_{2}\right)$ or $\left(-\phi_{2}\right)$.)

Lemma (3.6) comes from the following general result due to Haefliger.

Proposition 3.7 (HAEFLIGER). Let $M^{m}$ be a differentiable manifold, $Q^{m-1} \subseteq \partial M^{m}$ a submanifold of $\partial M^{m}$. Let both $Q^{m-1}$ and $M^{m}$ be r-connected, and assume $k$ is a positive integer so that $m \geqq 2 k-r+2,2 r<k$.

Then any two differentiable imbeddings

$$
\begin{aligned}
& \psi_{1}:\left(D^{k}, \partial D^{k}\right) \rightarrow\left(M^{m}, Q^{m-1}\right), \\
& \psi_{2}:\left(D^{k}, \partial D^{k}\right) \rightarrow\left(M^{m}, Q^{m-1}\right)
\end{aligned}
$$

which are homotopic, as continuous maps of pairs, are differentiably isotopic (I.e., there is a global automorphism $H:(M, Q) \rightarrow(M, Q)$ which is isotopic to the identity automorphisms such that $H \circ \psi_{1}=\psi_{2}$.) 
Proof. A generalization of the absolute version of the above appears reported in [2]; a proof (of a much more general theorem) is given in [3]. In [3], it is mentioned that a relative version is forthcoming in later papers, and an analysis of his techniques shows that they would succeed in proving Proposition (3.7) above.

To apply Proposition (3.7) in our case, we see that for $M=A_{2} \times D^{k}$, $Q=A_{2} \times \partial D^{k}, m=2 n+1, k=n+1, r=3, n \geqq 8$.

According to Lemma (3.6),

$$
\phi_{1} \sim \pm \phi_{2}
$$

and therefore Proposition (3.7) applies, concluding the proof of Lemma (3.6).

LEMMA 3.8. There is a diffeomorphism

$$
\psi:\left(A_{1} \times D^{k}, A_{1} \times \partial D^{k}\right) \underset{\approx}{\underset{\approx}{(}}\left(A_{2} \times D^{k}, A_{2} \times \partial D^{k}\right)
$$

such that

$$
\psi\left(a_{1} \times D^{k}\right)=a_{2} \times D^{k}
$$

and $\psi$ is homotopic to $f$.

Proof. Take $\psi=\mathrm{H}_{2} \circ \phi$ where $\mathrm{H}_{2}$ is the automorphism produced by Lemma (3.6). This is true for all $k \geqq n+1$ as follows: It has been proven that there is a diffeomorphism,

$$
\psi_{n+1}:\left(A_{1} \times D^{n+1}, A_{1} \times \partial D^{n+1}\right) \underset{\approx}{\underset{2}{a}}\left(A_{2} \times D^{n+1}, A_{2} \times \partial D^{n+1}\right)
$$

such that

$$
\psi_{n+1}\left(a_{1} \times D^{n+1}\right)=a_{2} \times D^{n+1} .
$$

For $k \geqq n+1$, let $\rho=k-n-1$, and define

$$
\psi_{k}: A_{1} \times D^{k} \approx\left(A_{1} \times D^{n+1}\right) \times D^{\rho} \stackrel{\psi_{n+1}}{ } \times 1
$$

Clearly $\psi_{k}$ has the desired properties. To study the homotopy properties of $\psi=\psi_{k}$, recall that $\psi$ induces a map

$$
\psi:\left(A_{1} \times D^{k}, A_{1} \times \partial D^{k}\right) \underset{\approx}{\underset{ }{2}}\left(A_{2} \times D^{k}, A_{2} \times \partial D^{k}\right)
$$

homotopic to $f \times 1_{k}$ (where $\psi, f \times 1_{k}$ are regarded as mappings of the above couple). This was guaranteed originally by the strong stability theorem. Our isotopies have not changed this.

To conclude the proof of Theorem (3.4) notice that $\psi$ induces a mapping

$$
\eta:\left(E^{k} A_{1}, \partial E^{k} A_{1}\right) \underset{\approx}{\underset{\approx}{*}}\left(E^{k} A_{2}, \partial E^{k} A_{2}\right)
$$

which is a diffeomorphism of the relative differentiable manifold pairs, 
$\left\{E^{k}\left(A_{i}\right),\left(\chi_{i}\right)_{k}\right\}, \quad i=1$, 2. Clearly $\eta \sim E^{k}(f)$ since $\psi \sim f \times 1_{k}$ on the pair $\left(A_{1} \times D^{k}, A_{1} \times \partial D^{k}\right)$.

4. A result concerning simple homotopy types. I shall make use of a fact concerning the calculation of torsion invariants of simple homotopy theory.

If $X$ is a finite complex, for $\lambda \in K^{0}(X)$, I shall define a map, denoted:

$$
\lambda: X \times S^{q} \rightarrow X \times S^{q}
$$

for $q$ sufficiently large (i.e., $q>\operatorname{dim} X$ ). The continuous map $\lambda$ will be unique up to homotopy class.

Let $b_{\lambda}: X \rightarrow S O_{q+1}$ be a representative of $\lambda$; then

$$
\lambda(\chi, s)=\left(\chi, b_{\lambda}(\chi) \cdot s\right)
$$

where $s \in S^{q}=\partial D^{q+1} \subseteq R^{q+1}, \chi \in X$ and $b_{\lambda}(\chi) \cdot s$ refers to the image of $s \in R^{q+1}$ under the linear transformation $b_{\lambda}(\chi) \in S O_{q+1}$. Of course, $b_{\lambda}(\chi) \cdot s \in S^{q}$.

Proposition 4.1 (MILNOR). Let $X \rightarrow{ }^{f} Y$ be a homotopy equivalence between finite complexes. Let $q$ be an odd integer. Then the map

$$
\lambda \circ\left(f \times 1_{q}\right): X \times S^{q} \rightarrow Y \times S^{q}
$$

is a simple homotopy equivalence for any $\lambda \in K^{0}(Y)$.

I shall not give the proof of Proposition (4.1) here.

Note, however, that it suffices to prove that

$$
f \times 1_{q}: X \times S^{q} \rightarrow Y \times S^{q}
$$

is a simple homotopy equivalence since $\lambda$ is a combinatorial isomorphism. A strictly weaker result is proved in [8] and it makes use only of the following property of $S^{q}$ : its Euler characteristic, $X\left(S^{q}\right)$, is zero. In a manner similar to [8], the Whitehead torsion of $f \times 1_{q}$ may be computed and shown to be zero.

5. Lens spaces. For arbitrary pairs $(p, q)$ of relatively prime positive integers, $p>q$, one defines a complex linear representation of the cyclic group $\boldsymbol{Z}_{\boldsymbol{p}}$ on $\boldsymbol{C}^{2}=\left\{\left(z_{1}, z_{2}\right) \mid z_{i} \in \boldsymbol{C}\right\}$ as follows:

Let $\omega$ be the complex number, $\omega=\exp (2 \pi i / p)$. If $\zeta \in \boldsymbol{Z}_{p}$ is a generator of $\boldsymbol{Z}_{p}$, define:

$$
\alpha_{\zeta}\left(z_{1}, z_{2}\right)=\left(\omega z_{1}, \omega^{q} z_{2}\right) .
$$

This induces a linear action of $\boldsymbol{Z}_{\boldsymbol{p}}$ on $\boldsymbol{C}^{2}$, which is fixed-point free when restricted to

$$
S^{3}=\left\{\left.\left(z_{1}, z_{2}\right) \in C^{2}|| z_{1}\right|^{2}+\left|z_{2}\right|^{2}=1\right\} .
$$

The quotient manifold $S^{3} / Z_{p}$ is called the lens space $L(p, q)$. It is a well-known result of J. H. C. Whitehead that the two lens spaces $L_{1}=L(7,1), L_{2}=L(7,2)$ 
are of the same homotopy type, but of distinct simple homotopy type. (See [14]. For a historical survey of the classification theory of lens spaces, see [8].)

These manifolds $L_{1}, L_{2}$ are parallelizable (as are all orientable 3-manifolds) and they are imbeddable differentiably (and uniquely up to isotopy class) in $S^{n}$ for $n \geqq 7$. Their normal bundles with respect to these imbeddings are trivial and therefore $L_{i} \times S^{n}$ possesses a tubular neighborhood

$$
L_{i} \times D^{n-3} \subseteq S^{n}
$$

for $i=1,2$. Actually, by a recent result of Hirsch [4], the $L_{i}$ (more generally: all orientable 3-manifolds) are imbeddable in $S^{5}$ with trivial normal bundle.

Let $M_{i}^{n} \subseteq S^{n}$ be the bounded complement of $L_{i} \times D^{n-3}$ as imbedded in $S^{n}$ $(i=1,2)$. Then $M_{i}^{n}$ is parallelizable (since it is obtainable as the closure of an open submanifold of euclidean space), and $\partial M_{i}^{n}=L_{i} \times S^{n-4}=\partial\left(L_{i} \times D^{n-3}\right)$. For simplicity, let us choose a base point $m \in \operatorname{int} M_{1} \cap \operatorname{int} M_{2} \subset S^{n}$, common to $M_{1}$ and $M_{2}$.

If $n$ is chosen so that $n \geqq 8, M_{i}^{n}$ is 3 -connected.

If $n \geqq 7$, the imbedding

$$
L_{i} \times D^{n-3} \underset{\beta_{(i)}^{n}}{\longrightarrow} S^{n}
$$

(for $i=1,2$ ) may be regarded as coming from

$$
L_{i} \times D^{n-4} \underset{\beta_{(i)}^{n-1}}{\longrightarrow} S^{n-1}
$$

by "suspension":

$$
\beta_{(i)}^{n}: L_{i} \times D^{n-3}=L_{i} \times D^{n-4} \times I \underset{\beta_{i}^{n-1} \times 1}{\longrightarrow} S^{n-1} \times I \subseteq S^{n} .
$$

LEMMA 5.1. Let $n \equiv 1(2), n>8$. There exists a continuous map $\rho: S^{n} \rightarrow S^{n}$ such that $\rho:\left(S^{n}, L_{1} \times D^{n-3}, L_{1} \times \partial D^{n-3}\right) \rightarrow\left(S^{n}, L_{2} \times D^{n-3}, L_{2} \times \partial D^{n-3}\right)$ is a homotopy equivalence of the triples $\left(S^{n}, L_{i} \times D^{n-3}, L_{i} \times \partial D^{n-3}\right)(i=1,2)$, and $\rho: L_{1} \times \partial D^{n-3} \rightarrow L_{2} \times \partial D^{n-3}$ is a simple homotopy equivalence.

Proof. Let $\rho: S^{n} \rightarrow S^{n}$ be the continuous map,

$$
\rho:\left(S^{n}, L_{1} \times D^{n-3}, L_{1} \times \partial D^{n-3}\right) \rightarrow\left(S^{n}, L_{2} \times D^{n-3}, L_{2} \times \partial D^{n-3}\right)
$$

obtained in this manner:

Since $n$ is large enough, it is possible to alter $\beta_{1}: L_{1} \times D^{n-3} \rightarrow S^{n}$ by isotopy so that these inclusions hold:

$$
\begin{aligned}
\beta_{2}\left(L_{2} \times D_{(1 / 2)}^{n-3}\right) & \subseteq \text { int } \beta_{1}\left(L_{1} \times D^{n-3}\right), \\
\beta_{1}\left(L_{1} \times D^{n-3}\right) & \subseteq \text { int } \beta_{2}\left(L_{2} \times D^{n-3}\right)
\end{aligned}
$$


and furthermore, the inclusion

$$
\beta_{1}\left(L_{1} \times D^{n-3}\right) \subseteq \beta_{2}\left(L_{2} \times D^{n-3}\right)
$$

is "homotopic" to the original homotopy equivalence $f: L_{1} \rightarrow L_{2}$.

Let $\rho: S^{n} \rightarrow S^{n}$ be the continuous map possessing these properties:

$(\rho)_{1}$ : The map $\rho \mid M_{2}^{n}$ is the identity map.

$(\rho)_{2}$ : The map $\rho \mid \beta_{2}\left(L_{2} \times D_{(1 / 2)}^{n-3}\right)$ is scalar-multiplication by 2 in the trivial linear disc-bundle, $L_{2} \times D^{n-3}$. (I.e., $\rho=\beta_{2} \circ 2 \circ \beta_{2}^{-1}$, where

$$
2: L_{2} \times D_{(1 / 2)}^{n-3} \rightarrow L_{2} \times D^{n-3}
$$

is defined by $2(\chi, d)=(\chi, 2 d)$.)

$(\rho)_{3}$ : If $\chi \in \beta_{2}\left(L_{2} \times D^{n-3}\right)-\beta_{2}\left(L_{2} \times D_{(1 / 2)}^{n-3}\right)$, then

$$
\rho(\chi)=\beta_{2}\left(\beta_{2}^{-1} \chi /\left\|\beta_{2}^{-1} \chi\right\|\right) \text {. }
$$

(The map $\rho$ simply "squeezes"

$$
\beta_{2}\left(L_{2} \times D^{n-3}\right)-\beta_{2}\left(L_{2} \times D_{(1 / 2)}^{n-3}\right)
$$

onto $\beta_{2}\left(L_{2} \times \partial D^{n-3}\right)$.)

LEMMA 5.2. The mapping

$$
\rho:\left(L_{1} \times D^{n-3}, L_{1} \times \partial D^{n-3}\right) \rightarrow\left(L_{2} \times D^{n-3}, L_{2} \times \partial D^{n-3}\right)
$$

is homotopic to $\lambda \circ\left(f \times 1_{n-3}\right)$, for some $\lambda \in K^{0}\left(L_{2}\right)$.

(Recall that for any complex $X$, for each $\lambda \in K^{0}(X)$ there is a mapping, denoted

$$
\lambda: X \times\left(D^{q}, \partial D^{q}\right) \rightarrow X \times\left(D^{q}, \partial D^{q}\right),
$$

uniquely given (up to homotopy) by: $\lambda(\chi, d)=b_{\lambda}(\chi) \cdot d\left(\chi \in X, d \in D^{q}\right)$ where $b_{\lambda}: X \rightarrow S O_{q}$ is a representative of the class $\lambda$ for $q$ sufficiently large.)

Since $L_{1} \sim L_{2}$, and this question is entirely a homotopy-theoretic matter, suppress the index $i$, and let $L=L_{1} \sim L_{2}$. Let $q=n-3$, and assume given

$$
\rho: L \times\left(D^{q}, \partial D^{q}\right) \rightarrow L \times\left(D^{q}, \partial D^{q}\right),
$$

a homotopy equivalence such that

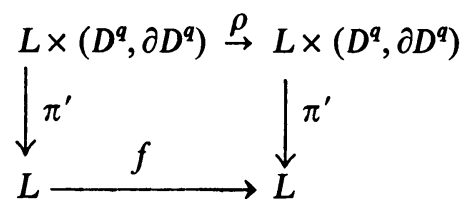

is homotopy commutative.

Let us prove that there is a $\lambda \in K^{0}(L)$ such that $\rho \sim \lambda \circ\left(f \times 1_{q}\right)$.

Letting 


$$
L \times\left(D^{q}, \partial D^{q}\right) \stackrel{\pi}{\longrightarrow}\left(D^{q}, \partial D^{q}\right)
$$

$L$

be the natural projections, it suffices to prove that the projections $\rho^{\prime}=\pi^{\prime} \rho, \rho^{\prime \prime}=\pi^{\prime \prime} \rho$ are homotopic to the projections of $\alpha=\lambda \circ\left(f \times 1_{q}\right)$. Homotopy commutativity of (5.3) insures that $\rho^{\prime} \sim \alpha^{\prime}$. It suffices to show $\rho^{\prime \prime} \sim \alpha^{\prime \prime}$.

Let $\Lambda_{q}$ be the function-space of all continuous maps

$$
s:\left(D^{q}, \partial D^{q}\right) \rightarrow\left(D^{q}, \partial D^{q}\right)
$$

which are homotopic to the identity (endowed with the $C-O$ topology.)

Let $d_{0} \in \partial D^{q} \subseteq D^{q}$ be a base point, and $\pi_{q}: \Lambda_{q} \rightarrow \partial D^{q}$ the evaluation map, $\pi_{q}(s)=s\left(d_{0}\right)$. If $\Omega_{q}=\left\{s \in \Lambda_{q} ; s\left(d_{0}\right)=d_{0}\right\}$, then the triple $\Omega_{q} \rightarrow \Lambda_{q} \rightarrow \partial D^{q}$ is a Serre fibre space. If $\lambda \in S O_{q}$ is a linear rotation of $R^{q}, \lambda$ restricts to a map, $\lambda:\left(D^{q}, \partial D^{q}\right) \rightarrow\left(D^{q}, \partial D^{q}\right)$.

Let $S O_{q-1} \subseteq S O_{q}$ be the subgroup leaving $d_{0}$ fixed. Then $S O_{q-1}$ may be regarded as a subspace of $\Omega_{q}$.

$$
S O_{q-1} \stackrel{j_{q}}{\rightarrow} \Omega_{q}
$$

It is easily seen that $\Omega_{q} \sim \Omega^{q-1}\left(\partial D^{q}\right)$ (where $\Omega^{p} X$ is the $p$-fold loop space of $X$ ).

The map

$$
\rho^{\prime \prime}: L \times\left(D^{q}, \partial D^{q}\right) \rightarrow\left(D^{q}, \partial D^{q}\right)
$$

induces a mapping

$$
g: L \rightarrow \Lambda_{q}
$$

where $g(l)(d)=\rho^{\prime \prime}(l, d)$.

To prove (5.2), it suffices to show the existence of a map

$$
g_{\lambda}: L \rightarrow S O_{q-1}
$$

( $g_{\lambda}$, a representative for some $\lambda \in K^{0}(L)$ ) such that

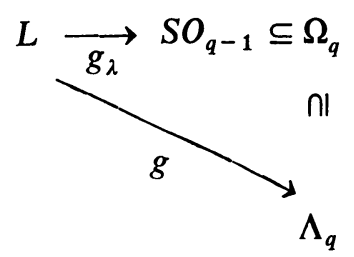

is homotopy commutative.

Since $\operatorname{dim} L=3, q-1=n-4 \geqq 4$, so $\partial D^{q}$ is 3 -connected. Therefore the map $\pi_{q} \circ g: L \rightarrow \partial D^{q}$ is homotopic to zero. Since 


$$
\Omega_{q} \rightarrow \Lambda_{q} \rightarrow \partial D^{q}
$$

is a Serre fibre space, there is a $g^{\prime}: L \rightarrow \Omega_{q}$ such that

is homotopy commutative.

$$
\stackrel{g^{\prime}}{\longrightarrow} \Omega_{q}
$$

Notice that $L$ may be given, up to homotopy type, by the following $C W$ description [8]:

$$
L \sim S^{1} \cup_{7} D^{2} \cup D^{3}
$$

Thus $g^{\prime}: L \rightarrow \Omega_{q}$ restricts to a mapping

$$
g_{2}^{\prime}: S^{1} \cup_{7} D^{2} \rightarrow \Omega_{q} \text {. }
$$

Since

$$
\begin{aligned}
& \pi_{1}\left(\Omega_{q}\right)=\pi_{q}\left(S^{q-1}\right) \approx Z_{2} \\
& \pi_{2}\left(\Omega_{q}\right)=\pi_{q+1}\left(S^{q-1}\right) \approx Z_{2}
\end{aligned}
$$

and

$$
H^{j}\left(S^{\prime} \cup{ }_{7} D^{2}, Z_{2}\right)=0, \quad j>0,
$$

it follows by obstruction theory that any mapping

$$
g_{2}^{\prime}: S^{\prime} \cup{ }_{7} D^{2} \rightarrow \Omega_{q}
$$

is homotopic to zero. Since $L / S^{1} \cup{ }_{7} D^{2}=S^{3}$, the mapping $g^{\prime}: L \rightarrow \Omega_{q}$ factors (up to homotopy):

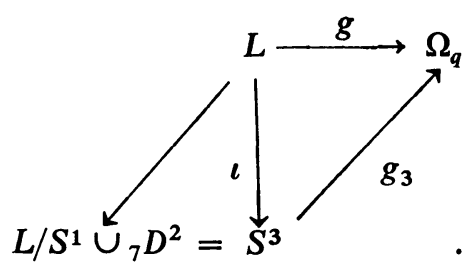

It is a classical computation (see Milnor-Kervaire, Bernoulli numbers, homotopy groups and a theorem of Rohlin, Proc. Internat. Congress of Math., Edinburgh, 1958) that

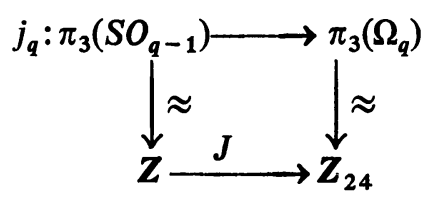

is surjective (for $q \geqq 5$ ). 
Therefore, there is a mapping $\tilde{g}_{3}: S^{3} \rightarrow S O_{q-1}$ such that

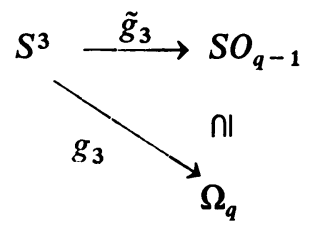

is homotopy commutative. Define $\tilde{g}: L \rightarrow S O_{q-1}$ by $\tilde{g}=\tilde{g}_{3} \circ \iota$ and the diagram

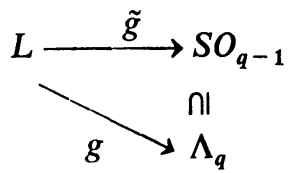

is homotopy commutative. Therefore $g \sim \tilde{g} \sim g_{\lambda}$ for $\lambda \in K^{0}(L)$ as was to be proved.

By Lemma (5.2),

$$
\partial \rho \sim \lambda \circ\left(f \times \partial 1_{n-3}\right): L_{1} \times \partial D^{n-3} \rightarrow L_{2} \times \partial D^{n-3} .
$$

Since $n-4 \equiv 1(2)$, Proposition (4.1) applies, giving us that

$$
\partial \rho: L_{1} \times \partial D^{n-3} \rightarrow L_{2} \times \partial D^{n-3}
$$

is a simple homotopy equivalence. This proves (5.1).

Notice that $\rho$ induces a map

$$
\rho:\left(M_{1}^{n}, \partial M_{1}^{n}\right) \rightarrow\left(M_{2}^{n}, \partial M_{2}^{n}\right)
$$

which is a homotopy equivalence of the pairs $\left(M_{i}, \partial M_{i}\right)$ and a simple homotopy equivalence between $\partial M_{i}$. Since the $M_{i}$ are 3-connected (simply connected will do), any homotopy equivalence is a simple homotopy equivalence (J. H. C. Whitehead [14]). Therefore

$$
\rho:\left(M_{1}, \partial M_{1}\right) \rightarrow\left(M_{2}, \partial M_{2}\right)
$$

is a simple homotopy equivalence between the manifold pairs $\left(M_{i}, \partial M_{i}\right)$. Since the $M_{i}^{n}$ are parallelizable, the map

$$
\rho:\left(M_{1}, \partial M_{1}\right) \rightarrow\left(M_{2}, \partial M_{2}\right)
$$

is a simple tangential equivalence. The map $\rho: S^{n} \rightarrow S^{n}$ also restricts to a homotopy equivalence,

$$
\rho:\left(L_{1} \times D^{n-3}, \partial\left(L_{1} \times D^{n-3}\right)\right) \rightarrow\left(L_{2} \times D^{n-3}, \partial\left(L_{2} \times D^{n-3}\right)\right)
$$

which is a tangential equivalence because the $L_{i} \times D^{n-3}$ are parallelizable.

Consider the inclusion maps,

$$
\sigma_{i}: E^{k} M_{i}^{n} \subseteq E^{k} S^{n} \approx S^{n+k} \quad(i=1,2)
$$


The $E^{k} M_{i}^{n}$ and $E^{k} S^{n}$ are taken with respect to the common base point $m$. The imbeddings $\sigma_{i}: E^{k} M_{i}^{n} \rightarrow S^{n+k}$ are relative diffeomorphisms.

LEMMA 5.5. There are differentiable imbeddings

$$
\varepsilon_{i}:\left(L_{i} \times D^{n-3}\right) \times R^{k} \rightarrow S^{n+k}
$$

for $i=1,2$ such that:

(1) $\sigma_{i}\left\{E^{k} M_{i}^{n}\right\} \cup \varepsilon_{i}\left\{\left(L_{i} \times D^{n-3}\right) \times R^{k}\right\}=S^{n+k}$,

(2) $\sigma_{i}\left\{E^{k} M_{i}^{n}\right\} \cap \varepsilon_{i}\left\{\left(L_{i} \times D^{n-3}\right) \times R^{k}\right\}=\varepsilon_{i}\left\{\partial\left(L_{i} \times D^{n-3}\right) \times R^{k}\right\}$.

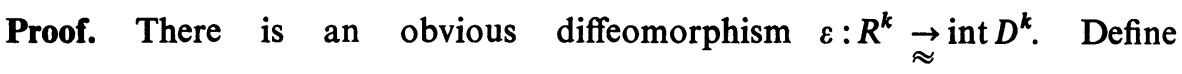

$$
\varepsilon_{i}:\left(L_{i} \times D^{n-3}\right) \times R^{k} \rightarrow E^{k}\left(S^{n}, m_{i}\right) \approx S^{n+k}
$$

to be the composite

$$
\left(L_{i} \times D^{n-3}\right) \times R^{k} \underset{\beta_{(i)}^{n} \times \varepsilon}{\longrightarrow} S^{n} \times D^{k} \underset{q_{i}}{\longrightarrow} E^{k}\left(S^{n}, m_{i}\right) \approx S^{n+k}
$$

where

$$
q: S^{n} \times D^{k} \rightarrow\left(S^{n} \times D^{k} /\left\{m_{i}\right\} \times D^{k} \cup S^{n} \times \partial D^{k}\right)=E^{k}\left(S^{n}, m_{i}\right)
$$

is the natural identification. Properties (1) and (2) are evident.

Recall that $n$ is subject to the conditions: $n \equiv 1(\bmod 2), n>8$. Set $k=n+1$. Let $K_{i}=E^{k} M_{i}^{n}, i=1,2$.

Since the $M_{i}^{n}$ are 3-connected, and $\rho: S^{n} \rightarrow S^{n}$ induces a simple tangential equivalence,

$$
\rho:\left(M_{1}^{n}, \partial M_{1}^{n}\right) \rightarrow\left(M_{2}^{n}, \partial M_{2}^{n}\right),
$$

Theorem (3.4) applies, giving us a "diffeomorphism"

such that the diagram

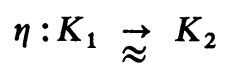

$$
\begin{array}{ccc}
K_{1} \stackrel{\eta}{\longrightarrow} & K_{2} \\
\| & \| \\
E^{k} M_{1}^{n} & E^{k} M_{2}^{n} \\
\text { ก। } & \text { ก। } \\
E^{k} S^{n} & E^{k} \rho & E^{k} S^{n}
\end{array}
$$

is homotopy commutative.

It is standard that one can find a continuous mapping

$$
\rho_{0}:\left(E^{k} S^{n}, K_{1}\right) \rightarrow\left(E^{k} S^{n}, K_{2}\right)
$$

possessing properties analogous to the properties of $\rho$ listed in (5.1) such that the diagram 


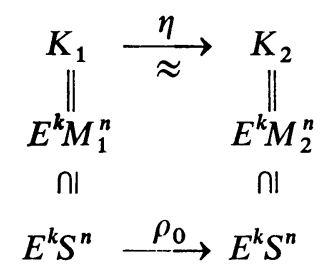

is actually commutative.

We may also insist of $\rho_{0}$ (thanks to a similar property of $\rho$ given in (5.1)) that there be a tangential homotopy equivalence

$$
\omega_{0}:\left(L_{1} \times D^{n-3}, \partial\left(L_{1} \times D^{n-3}\right)\right) \times R^{k} \rightarrow\left(L_{2} \times D^{n-3}, \partial\left(L_{2} \times D^{n-3}\right)\right) \times R^{k}
$$

such that

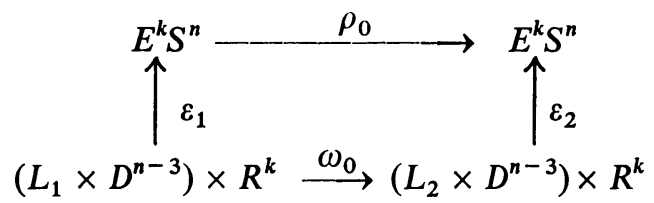

is commutative.

According to Lemma (5.5),

$$
\sigma_{i}\left(K_{i}\right) \cap \varepsilon_{i}\left(\left(L_{i} \times D^{n-3}\right) \times R^{k}\right)=\varepsilon_{i}\left\{\partial\left(L_{i} \times D^{n-3}\right) \times R^{k}\right\} .
$$

The following diagram, then, makes sense, and is commutative:

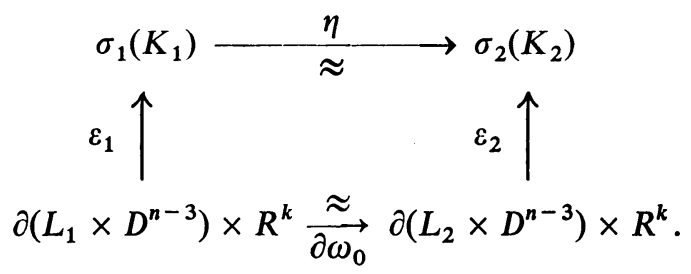

This shows that $\omega_{0}$, restricted to $\partial\left(L_{1} \times D^{n-3}\right) \times R^{k}$, is a diffeomorphism between $\partial\left(L_{1} \times D^{n-3}\right) \times R^{k}$ and $\partial\left(L_{2} \times D^{n-3}\right) \times R^{k}$.

Now recall the "action" $\Phi$, previously defined in $\S 3$, representing $K^{0}\left(M_{2}^{n}\right)$ as a group of "differentiable" automorphisms (unıque up to isotopy class) of $E^{k} M_{2}^{n}$. For each $\lambda \in K^{0}\left(M_{2}^{n}\right)$ one has an automorphism $\phi_{\lambda}: K_{2} \underset{\approx}{\vec{*}} K_{2}$.

Define (for each $\lambda \in K^{0}\left(M_{2}^{n}\right)$ )

$$
\eta_{\lambda}: K_{1} \underset{\approx}{\approx} K_{2}
$$

to be the composite,

$$
\eta_{\lambda}: K_{1} \underset{\approx}{\stackrel{\eta}{\approx}} K_{2} \underset{\approx}{\stackrel{\phi_{\lambda}}{\longrightarrow}} K_{2}
$$


Define $\partial \omega_{\lambda}: \partial\left(L_{1} \times D^{n-3}\right) \times R^{k} \rightarrow \partial\left(L_{2} \times D^{n-3}\right) \times R^{k}$ to be the map such that

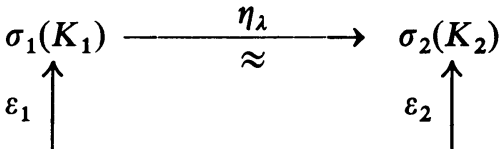

$$
\partial\left(L_{1} \times D^{n-3}\right) \times R^{k} \stackrel{\partial \omega_{\lambda}}{\underset{\approx}{\longrightarrow}} \partial\left(L_{2} \times D^{n-3}\right) \times R^{k}
$$

is commutative (i.e., $\partial \omega_{\lambda}=\varepsilon_{2}^{-1} \phi_{\lambda} \varepsilon_{2} \partial \omega_{0}$ ); one has the following lemma:

LEMMA 5.11. The map $\partial \omega_{\lambda}$ (for any $\lambda \in K^{0}\left(M_{2}^{n}\right)$ ) extends to a tangential homotopy equivalence

$$
\omega_{\lambda}:\left(L_{1} \times D^{n-3}, \partial\left(L_{1} \times D^{n-3}\right)\right) \times R^{k} \rightarrow\left(L_{2} \times D^{n-3}, \partial\left(L_{2} \times D^{n-3}\right)\right) \times R^{k} .
$$

Proof. The map $\partial \omega_{\lambda}$ is homotopic to $\partial \omega_{0}$. Since $\partial \omega_{0}$ extends to a tangential homotopy equivalence

$$
\omega_{0}:\left(L_{1} \times D^{n-3}, \partial\left(L_{1} \times D^{n-3}\right)\right) \times R^{k} \rightarrow\left(L_{2} \times D^{n-3}, \partial\left(L_{2} \times D^{n-3}\right)\right) \times R^{k},
$$

so does $\partial \omega_{\lambda}$.

REMARK. The tangential homotopy equivalence,

$$
\omega_{\lambda}:\left(L_{1} \times D^{n-3}, \partial\left(L_{1} \times D^{n-3}\right)\right) \times R^{k} \rightarrow\left(L_{2} \times D^{n-3}, \partial\left(L_{2} \times D^{n-3}\right)\right) \times R^{k}
$$

satisfies properties $\left(\partial_{1}\right)$ and $\left(\partial_{2}\right)$ of $\S 2$. Therefore $\omega_{\lambda}$ satisfies all the hypotheses of the weak extension theorem $(\S 2)$ except for $\left(\partial_{3}\right)$ which requires that the obstruction $k\left(\omega_{\lambda}\right) \in k\left(L_{2} \times D^{n-3}, \partial\left(L_{2} \times D^{n-3}\right)\right)$ vanish.

I now revert to the terminology of Proposition (1.1) of $\$ 1$. Set: $A=L_{2} \times D^{n-3}, B=M_{2}^{n}, W=\partial\left(L_{2} \times D^{n-3}\right)=\partial\left(M_{2}^{n}\right)$.

Define a map

$$
g: K^{0}(B) \rightarrow k(A, W)
$$

by $g(\lambda)=k\left(\omega_{\lambda}\right) \in k(A, W)$, for $\lambda \in K^{0}(B)$.

LEMMA 5.12. The map

$$
(g-\gamma): K^{0}(B) \rightarrow k(A, W)
$$

is a constant. (The $\gamma$ is defined in Proposition 1.1.)

Proof. Straightforward.

Notice that our particular $A, B, W$ satisfy the hypotheses of Proposition (1.1) if $n \equiv 3,5,7(\bmod 8)$ (i.e., $\left.A \cap B=W, A \cup B=S^{n}\right)$.

LEMMA 5.13. Let $n \equiv 3,5,7(\bmod 8)$ (as well as the standard requirement: $n>8)$.

Let $A, B, W$ be as above. Then the map $g: K^{0}(B) \rightarrow k(A, W)$ is surjective.

Since $n \equiv 3,5,7(\bmod 8)$, Proposition (1.1) applies, which yields surjectivity 
of $\gamma$. Since (by 5.12) $g-\gamma$ is a constant, it follows that the map $g$ is surjective as well. From now on I assume $n \equiv 3,5,7(\bmod 8), n>8$. By surjectivity of $g$, there is a $\lambda_{0} \in K^{0}\left(M_{2}^{n}\right)$ such that

$$
g\left(\lambda_{0}\right)=O \in k\left(L_{2} \times D^{n-3}, \partial\left(L_{2} \times D^{n-3}\right)\right) .
$$

Thus, $g\left(\lambda_{0}\right)=k\left(\omega_{\lambda_{0}}\right)=O$. Since $\omega_{\lambda_{0}}$ now satisfies the hypotheses $(\partial)$ of the weak extension theorem, we may conclude that there exists a diffeomorphism

$$
\left.\omega:\left(L_{1} \times D^{n-3}, \partial\left(L_{1} \times D^{n-3}\right)\right) \times R^{k} \underset{\approx}{(}\left(L_{2} \times D^{n-3}\right)\right) \times R^{k}
$$

so that

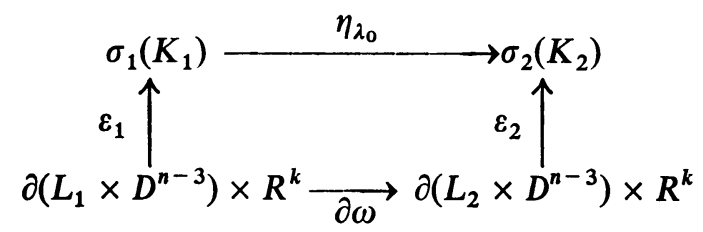

is commutative.

LEMMA 5.15. Let

$$
\omega:\left(L_{1} \times D^{n-3}, \partial\left(L_{1} \times D^{n-3}\right)\right) \times R^{k} \underset{\approx}{(}\left(L_{2} \times D^{n-3}, \partial\left(L_{2} \times D^{n-3}\right) \times R^{k}\right.
$$

be a diffeomorphism of manifold pairs. As usual, denote:

$$
\partial \omega=\omega \mid \partial\left(L_{1} \times D^{n-3}\right) \times R^{k} .
$$

Assume that (5.14) is commutative for such an $\omega$. Then there is a unique continuous map

$$
h: E^{k} S^{n} \rightarrow E^{k} S^{n}
$$

satisfying the commutative diagram

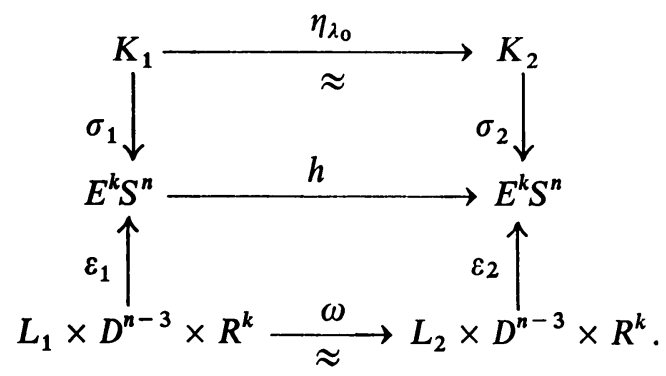

The map $h: E^{k} S^{n} \rightarrow E^{k} S^{n}$ is a topological homeomorphism.

Proof. Define $h$ so as to make (5.16) commutative. According to the decomposition of $E^{k} S^{n}=S^{n+k}$ given in Lemma 5.5, uniqueness is obvious. For 
existence of $h$, one must check compatibility of the two definitions of $h$ on $\varepsilon_{1}\left(L_{1} \times \partial D^{n-3}\right)$. This follows from the commutativity of (5.10).

One may check continuity of $h$ easily. The map $h$ is a topological homeomorphism because $h^{-1}$ may be constructed similarly from the diagram

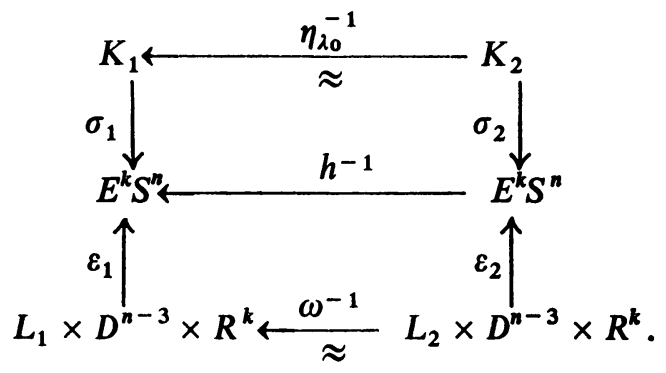

Thus, applying Lemma (5.15) to our situation, we may conclude that there exists a topological homeomorphism $h: S^{n+k} \rightarrow S^{n+k}$ such that

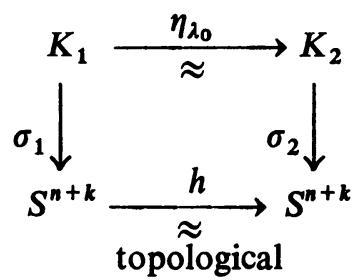

is commutative.

Now let $K$ be the finite complex $K_{1}$, and let

$$
\alpha: K \rightarrow S^{n+k}
$$

be the combinatorial imbedding of $K$ in $S^{n+k}$ given by

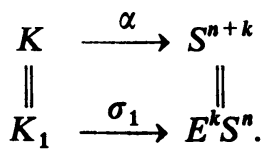

Let $\beta: K \rightarrow S^{n+k}$ be the combinatorial imbedding obtained as follows:

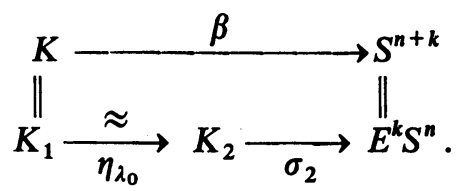

THEOREM. If $n>7$, the combinatorial imbeddings $\alpha, \beta: K \rightarrow S^{2 n+1}$ (can be defined and) are combinatorially inequivalent.

If, further,

$$
n \equiv 3,5,7(\bmod 8),
$$


then there is a topological homeomorphism $h: S^{2 n+1} \rightarrow S^{2 n+1}$ which establishes a topological equivalence between $\alpha$ and $\beta$. That is, the diagram

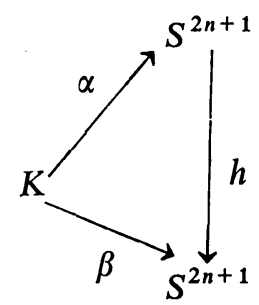

is commutative.

REMARK. The smallest dimension allowed by these restrictions on $n$ is: $\operatorname{dim} K=23$.

Proof. Let $X_{i}$ be the finite complexes (for $i=1,2$ ) obtained as follows:

$$
\begin{aligned}
& X_{1}=S^{n+k} / \alpha(K)=E^{k} S^{n} / K_{1}, \\
& X_{2}=S^{n+k} / \beta(K)=E^{k} S^{n} / K_{2}
\end{aligned}
$$

(cf. Lemma (0.1)). It is then obvious (by the definition of $E^{k} S^{n}$ ) that

$$
X_{i} \approx L_{i} \times D^{n-3} \times D^{k} \cup \mathrm{C}\left\{\partial\left(L_{i} \times D^{n-3} \times D^{k}\right)\right\} \quad \text { for } i=1,2
$$

where $\mathrm{CX}$ is the cone over $X$, and the union of the two spaces in (5.19) is made by identifying

$$
\partial\left(L_{i} \times D^{n-3} \times D^{k}\right) \subseteq \mathrm{C}\left\{\partial\left(L_{i} \times D^{n-3} \times D^{k}\right)\right\}
$$

with

$$
\partial\left(L_{i} \times D^{n-3} \times D^{k}\right) \subseteq L_{i} \times D^{n-3} \times D^{k}
$$

by the identity map.

Therefore it is clear that our spaces $X_{1}$ and $X_{2}$ are precisely Milnor's spaces $X_{1}$ and $X_{2}$ in [8] (see p. 575). Milnor's Theorem 2 applies, giving us that $X_{1}$ and $X_{2}$ are combinatorially inequivalent. Clearly a combinatorial equivalence between $\alpha$ and $\beta$ would induce a combinatorial equivalence between $X_{1}$ and $X_{2}$. The efore we may conclude that there does not exist a combinatorial auto morphism

such that

$$
\kappa: S^{2 n+1} \rightarrow S^{2 n+1}
$$

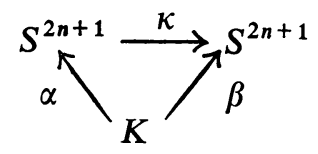


is commutative. (More is actually proved. There does not exist a combinatorial

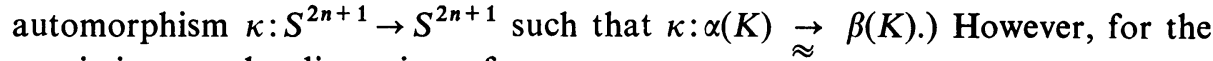
restriction on the dimension of $n$,

$$
n \equiv 3,5,7(\bmod 8)
$$

the topological homeomorphism

$$
h: S^{2 n+1} \rightarrow S^{2 n+1}
$$

has already been constructed such that (5.17) is commutative. It then follows by the definitions of $\alpha, \beta$ that

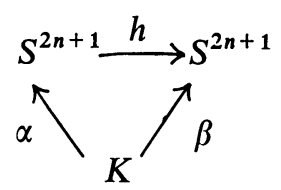

is commutative. This concludes the proof of the theorem.

6. Topological equivalences of general combinatorial imbeddings. In this section I shall abstract a more general situation in which one may say that two combinatorial imbeddings $\alpha, \beta: K \rightarrow S^{m}$ are topologically equivalent. The proof of the existence of the topological equivalence is quite clearly the same as the construction of the topological equivalence $h$ in $\$ 5$.

THEOREM 6.1. Let $n \equiv 3,5,6,7(\bmod 8), n \geqq 8$. Let $M_{i}^{n} \subseteq S_{i}^{n}(i=1,2)$ be bounded differentiable compact 3-connected $n$-dimensional submanifolds of $S_{i}^{n}$ (differentiable manifolds without boundary).

Let $N_{i}^{n}$ be the bounded complement of $M_{i}^{n}$ in $S_{i}^{n}$,

$$
M_{i}^{n} \cap N_{i}^{n}=W_{i}^{n-1}=\partial M_{i}^{n}=\partial N_{i}^{n} \quad(i=1,2) .
$$

Let $\rho: S_{1}^{n} \rightarrow S_{2}^{n}$ be a continuous map such that

(a) $\rho$ induces a map of couples

$$
\rho:\left(M_{1}^{n}, W_{1}^{n-1}\right) \rightarrow\left(M_{2}^{n}, W_{2}^{n-1}\right)
$$

which is a simple homotopy equivalence.

(b) $\rho$ induces a map of couples

$$
\rho:\left(N_{1}^{n}, W_{1}^{n-1}\right) \rightarrow\left(N_{2}^{n}, W_{2}^{n-1}\right)
$$

which is a homotopy equivalence.

Let $k \geqq n+1$. (Then (of course)

$$
E^{k} M_{i}^{n} \subseteq E^{k} S_{i}^{n} \quad(i=1,2)
$$

are finite subcomplexes.) 
Then:

There exists a topological homeomorphism

such that $h$ induces a map

$$
h: E^{k} S_{1}^{n} \rightarrow E^{k} S_{2}^{n}
$$

$$
h:\left(E^{k} M_{1}^{n}, \partial E^{k} M_{1}^{n}\right) \underset{\approx}{\approx}\left(E^{k} M_{2}^{n}, \partial E^{k} M_{2}^{n}\right)
$$

which is a combinatorial isomorphism. (Actually $h$ is a "diffeomorphismexcept-at-one-point.')

\section{REFERENCES}

1. R. Bott, The stable homotopy of the classical groups, Ann. of Math. (2) 70 (1959), 313-337.

2. A. Haefliger, Differentiable imbeddings, Bull. Amer. Math. Soc. 67 (1961), 109-112.

3. - Plongements différentiables de variétés dans variétés, Comment. Math. Helv. 36 (1961), 47-82.

4. M. Hirsch, The imbeddings of bounding manifolds in Euclidean space, Ann. of Math. (2) 74 (1961), 494-497.

5. S. Lang, Stable equivalence, Séminaire Bourbaki 13 (1960/61) (to appear).

6. B. Mazur, Stable equivalence of differentiable manifolds, Bull. Amer. Math. Soc. 67 (1961), 377-384.

7. - The theory of neighborhoods, (mimeographed), Harvard Univ., Cambridge, Mass., 1961.

8. J. Milnor, Two complexes which are homeomorphic but combinatorially distinct, Ann. of Math. (2) 74 (1961), 575-590.

9. —_, Lecture notes on differential topology, (mimeographed), Princeton Univ., Princeton, N. J., 1961.

10. E. Moise, Affine structures on 3-manifolds. V, Ann. of Math. (2) 56 (1952), 96-114.

11. C. Papakyriakopoulos, A new proof of the invariance of the homology groups of a complex, Bull. Soc. Math. Greece 22 (1943), 1-154. (Greek)

12. S. Smale, Differentiable and combinatorial structures on manifolds, Ann. of Math. (2) 74 (1961), 498-502.

13. J. Stallings, The topology of high-dimensional piece-wise linear manifolds, (mimeographed), Princeton Univ., Princeton, N. J., 1961.

14. J. H. C. Whitehead, Simple homotopy types, Amer. J. Math. 72 (1950), 1-57.

15. - On $C^{i}$-complexes, Ann. of Math. (2) 41 (1940), 809-824.

16. H. Whitney, Differentiable manifolds, Ann. of Math. (2) 37 (1936), 645-680.

17. E. C. Zeeman, Knotting manifolds, Bull. Amer. Math. Soc. 67 (1961), 117-119.

18. E. H. Connell, Approximating stable homeomorphisms, Bull. Amer. Math. Soc. 69 (1963), 87-90.

HARVARD UNIVERSITY,

CAmbridge, Massachusetts 